\title{
Development and characterization of dilutable self-microemulsifying premicroemulsion systems (SMEPMS) as templates for preparation of nanosized particulates
}

\author{
This article was published in the following Dove Press journal: \\ International Journal of Nanomedicine \\ 10 September 2013 \\ Number of times this article has been viewed
}

\author{
Shen-Fu Lin' \\ Ying-Chen Chen ${ }^{2}$ \\ $\mathrm{Hsiu}-\mathrm{O} \mathrm{\textrm {Ho } ^ { 2 }}$ \\ Wei-Yu Huang ${ }^{2}$ \\ Ming-Thau Sheu 2,3 \\ Der-Zen Liu',4 \\ 'Graduate Institute of Biomedical \\ Materials and Tissue Engineering, \\ College of Oral Medicine, ${ }^{2}$ School \\ of Pharmacy, College of Pharmacy, \\ Taipei Medical University, Taipei, \\ Taiwan; ${ }^{3}$ Clinical Research Center and \\ Traditional Herbal Medicine Research \\ Center, Taipei Medical University \\ Hospital, Taipei, Taiwan; ${ }^{4}$ Center for \\ General Education, Hsuan Chuang \\ University, Hsinchu, Taiwan
}

\begin{abstract}
The utilization of self-microemulsifying premicroemulsion systems (SMEPMS) as templates for preparing poorly water-soluble compounds in the nanosized range represents a promising strategy. Fenofibrate was formulated with n-butyl L-lactate, Tween 80 , and a number of cosurfactants (ethanol, 1-propanol, and PEG 600), diluted with the water phase (either water or saccharide solution) and then subjected to a freeze-drying (FD) process to obtain SMEPMS nanosized particulates. Results demonstrated that the particle size after resuspension of these FD SMEPMS nanosized particulates in water was too large, so the addition of saccharide solutions (lactose, mannitol, glucose, sucrose, and trehalose) as the solid carrier to prevent particles from aggregating seemed to be necessary and workable due to steric hindrance and repulsion. However, instability of these resuspended FD nanosized particulates after 30-90 minutes still occurred, and the addition of $0.5 \%$ sodium lauryl sulfate in the resuspending medium was able to retard the aggregation and maintain the particle size within the nano-range. Evaluation by scanning electron microscopy and X-ray powder diffraction also confirmed the results. It was concluded that using an SMEPMS formulation with PEG 600 as the cosurfactant, and in the presence of a suitable saccharide as an anticaking agent and FD process were able to produce fenofibrate nanoparticles.
\end{abstract}

Keywords: fenofibrate, saccharides, freeze-drying, nanoparticles

\section{Introduction}

Many newly discovered or existing drug molecules are poorly water-soluble, and thus, they face challenges to become successful therapies and to be marketed ${ }^{1}$ due to problems of poor bioavailability after oral administration. Since dissolution is the rate-determining step to make these drugs available to patients, formulation design is a practical approach to improve the absorption and efficacy of such drugs. ${ }^{2}$ Fenofibrate (FFB) is a neutral lipophilic compound $(\log P=5.24)$, and it is practically insoluble in water (with an aqueous solubility $<0.5 \mathrm{mg} / \mathrm{L}$ ), resulting in incomplete and irregular bioavailability after oral ingestion. Reduction in the particle size of FFB by a micronization process improved its solubility and subsequently increased the bioavailability. ${ }^{3}$ Therefore, development of a cost-effective process to reduce the particle size of FFB to improve its bioavailability would be beneficial.

Microemulsions (MEs) were first introduced decades ago by Hoar and Schulman ${ }^{4}$ and many advantages of MEs were found, especially in enhancing the oral bioavailability of poorly soluble drugs and nutraceuticals. ${ }^{5}$ Self-microemulsifying premicroemulsion 
systems (SMEPMS) are mixtures of oils and surfactants, sometimes including cosurfactants, and represent a promising alternative to poorly water-soluble compounds. SMEPMS can be self-emulsified into a single optically isotropic and thermodynamically stable ME when contacting with an aqueous medium under gentle digestive motility in the gastrointestinal tract. ${ }^{6-8}$ The intrinsic physicochemical properties include nanosize, transparency, low viscosity, and thermodynamic stability. ${ }^{8}$ Nanosized droplets have very high surface-to-volume ratios that lead to high solubilization capacities, enhanced drug release, and subsequently improved bioavailability, ${ }^{9}$ and offer release in a more reproducible manner, which is less dependent on the gastrointestinal physiology and the fed/ fasted state of patients. ${ }^{10}$

SMEPMS have been reported to improve the in vivo dissolution, and, therefore, enhance the bioavailability of lipophilic drugs. ${ }^{11-15}$ Commercially available drugs, including cyclosporin $\mathrm{A},{ }^{16}$ ritonavir, and saquinavir (HIV protease inhibitors), ${ }^{17}$ are formulated with SMEPMS, and the usefulness of these systems was also clinically demonstrated. However, SMEPMS usually possess liquids or is encapsulated in gelatin capsules. Such dosage forms might be inconvenient for patients' use and also tend to leak during storage. Hence, a strategy of incorporating liquid SMEPMS into a solid dosage form would overcome the shortcomings of liquid formulations. ${ }^{18}$

Furthermore, the concept of diluting ME systems with water (continuous phase) to produce nanoparticles of mitotane and griseofulvin by a diffusion technique was introduced by Trotta et al. ${ }^{19,20}$ The process is based on the water miscibility of those solvents used as the oil phase to solubilize the drug. Upon transferring a transient oil-inwater emulsion (E) or ME into water, the drug dissolved in the organic phase and instantly solidified due to the almost complete diffusion of the organic solvent from the droplets to the continuous phase. Using optimized formulations of Es or MEs and homogenization parameters, drug particles of $<100 \mathrm{~nm}$ with very narrow polydispersity and high dissolution were obtained. The feasibility of preparing nanodrugs of griseofulvin and mitotane from solvent-in-water MEs by the diffusion technique was validated using nontoxic solvents, surfactants, and cosurfactants. This indicated that optimized dilutable SMEPMS using these partially water-miscible solvents were able to be used as a template for preparing nanodrugs and nanoparticles.

One important application of ME systems is the use as templates, allowing one to obtain monodispersed sizes of nanoparticles (inorganic or organic) by varying the size of the
ME droplet radius. In this study, the utilization of dilutable SMEPMS to form ME as a template for preparing nanosized FFB was developed using n-butyl L-lactate, Tween 80, and a number of cosurfactants (ethanol, 1-propanol, and polyethylene glycol 600). Pseudo-ternary phase diagrams for dilutable SMEPMS were constructed to characterize and optimize the oil phase, surfactants, and cosurfactants to form FFB nanoparticulates.

\section{Materials and methods Materials}

n-Butyl L-lactate was purchased from Alfa Aesar GmbH and Co KG (Karlsruhe, Germany); the Tween series and 1-propanol were supplied by Riedel-de Haën (Seelze, Germany); ethanol was bought from Taiwan Sugar Corporation (Taipei, Taiwan); Cremophor RH-40 was purchased from BASF (Ludwigshafen, Germany); polyethylene glycol (PEG) 600 was supplied by Merck KGaA (Darmstadt, Germany); lactose and mannitol were from DFE Pharma (Borculo, the Netherlands); and $\mathrm{D}(+)$-glucose, sucrose, and $\mathrm{D}(+)$-trehalose were purchased from Sigma-Aldrich (St Louis, MO, USA)

\section{Construction of pseudo-ternary phase diagrams to form FFB SMEPMS}

The pseudo-ternary phase diagrams of oil, surfactant, and cosurfactant were developed using a water titration method. For each phase diagram, surfactant/cosurfactant (Tween 80/ ethanol, 1-propanol, PEG 600), at a specific ratio of 1/1, 1/4, 4/1 (w/w), and homogenous mixture of n-butyl L-lactate with/ without FFB was mixed by magnetic stirring to find the optimal type of cosurfactant and the ratio of surfactant/cosurfactant. Next, FFB was dissolved in n-butyl L-lactate, and surfactant/ cosurfactant was mixed at a specific ratio; oil phase and surfactant/cosurfactant were accurately weighed (from 1:9 to 9:1) into glass vials. Then, the components were mixed by gentle stirring and vortex mixing, diluted with 500 times the volume of water in a dropwise manner, and visually observed for phase clarity and flow ability. After the identification of $\mathrm{ME}$ region in the phase diagrams, the ME formulations were selected at desired component ratios. The same pseudo-ternary phase diagrams of SMEPMS with the oil phase saturated with $50 \%$ or $100 \%$ of FFB were also constructed.

\section{In situ formation and characterizations of FFB nanoparticles}

One gram of optimal formulations selected from SMEPMS regions (oil phase saturated with FFB) was added dropwise into $10 \mathrm{~mL}$ of various saccharide solutions (lactose, mannitol, 
glucose, sucrose, and trehalose, either in a concentration of 5\% or $10 \%$ ) at a rate of $3.3 \mathrm{~mL} /$ minute. After addition, the mixture was vortexed for 3 minutes and allowed to sit for another 30 minutes, and $1 \mathrm{~mL}$ of mixture was withdrawn to measure the size and distribution of particles (N5 Submicron Particle Size Analyzer; Beckman Coulter, Brea, CA, USA) formed in situ (ie, particle sizes before freeze-drying [BFD]). The N5 Submicron Particle Size Analyzer uses Photon Correlation Spectroscopy (PCS), which determines particle size by measuring the rate of fluctuations in laser light intensity scattered by particles as they diffuse through a fluid. Following the same procedure as previously described, particle sizes of the five formulations without/with saturation of the oil phase with FFB after diluting with water were also measured for comparison (designated as the blank and control, respectively). After the measurement, the rest mixture was sonicated for 10 minutes and then freezedried (FD) for at least 24 hours. The morphology of the FD powder was observed by scanning electron microscopy (SEM S-2400; Hitachi Ltd, Tokyo, Japan). The specimens were first gold-coated using a sputter coater (Hitachi IB-2; Hitachi) prior to SEM. The crystallinity of the FD powder was characterized by an X-ray powder diffraction method (Powder X-ray diffractometer MiniFlex; Rigaku Corporation, Tokyo, Japan). X-ray diffraction allows one to know if the sample is crystallized. The particle size and distribution of the FD powder were also measured after being reconstituted for 30 and 90 minutes (designated AFD30 $\mathrm{m}$ and AFD90 m, respectively) in either an aqueous solution or a $0.5 \% \mathrm{w} / \mathrm{w}$ sodium lauryl sulfate (SLS) solution of 50 times its weight. The transition time (TT) was designated as the time elapsed for the formation of turbidity (appearing when the particle size exceeded $250 \mathrm{~nm}$ ) after reconstituting the FD powder in an aqueous solution or a $0.5 \% \mathrm{w} / \mathrm{w}$ SLS solution. Figure 1 provides the scheme of SMEPMS preparation and characterizations.

\section{Statistical analysis}

All data were presented as mean \pm standard deviation. The statistical significance of differences was performed using analysis of variance (ANOVA) test by SPSS statistic software (PASW Statistics 18.0; IBM Corporation, Armonk, NY, USA). Difference was considered significant when the $P$-value was less than 0.05 .

\section{Results and discussion Construction of pseudo-ternary phase diagrams}

The solubility of FFB in n-butyl L-lactate was about $0.2 \mathrm{~g} / \mathrm{g}$. The oral $50 \%$ lethal dose of n-butyl L-lactate is $>5000 \mathrm{mg} /$ $\mathrm{kg}$ in rats, ${ }^{21}$ so it is considered as a safe oil phase in this study. A pseudo-ternary phase diagram of oil (n-butyl L-lactate)/ surfactant (Tween 80)/cosurfactant (ethanol, 1-propanol, and PEG 600) with/without saturation of FFB in the oil phase was constructed to determine the SMEPMS region. Surfactant possesses polar and nonpolar portions, and when placed in an oil-water system, the polar groups are attracted to or orient toward the water, and the nonpolar groups are oriented toward the oil. The surfactant molecule lowers the interfacial tension between the oil and water phases so that spontaneous dispersion of water or oil droplets occurs and the system is thermodynamically stable. An effective way to further decrease interfacial tension is to include a second surface-active species (either a surfactant or medium-chain alcohol) that is a cosurfactant. Tween and PEG series, 1-propanol, and ethanol have been previously reported as either effective surfactants or cosurfactants. ${ }^{11,22-25}$ For example, Wei et $\mathrm{a}^{22}$ found that the use of Tween 80 with $40 \%$ content had minimum self-emulsification time. Patel and Vavia $^{23}$ developed the optimized formulation of fenofibrate for in vitro dissolution and pharmacodynamic studies that was composed of Labrafac ${ }^{\circledR}$ CM10 (Gattefosse, Mumbai, India) (31.5\%), Tween 80 (47.3\%), and PEG 400 (12.7\%). Results are shown in Figure 2A-C and Figure 2D-F without/ with FFB saturation in the oil phase, respectively. Without FFB saturation in the oil phase, the SMEPMS (ie, ME; black area) regions for these three cosurfactants were all located at the bottom of the respective ternary diagrams and had similar areas. There was a region in which an $\mathrm{E}$ formed close to the corner of the oil phase when using PEG 600 as the cosurfactant. When the oil phase was saturated with FFB, the areas of the SMEPMS significantly decreased compared to that without FFB saturation for all these three cosurfactants, and some of them formed an E (gray area) system leading to an increase in the gray area. This phenomenon might be caused by the decreased interfacial tension between the oil phase and aqueous phase as a result of the solubilization of the hydrophobic FFB in the oil phase. Then the thermodynamic stability of the interface was affected according to Gibbs' equation. The presence of FFB molecules in the interface affected the structure of the micelles and raised the free energy.

SMEPMS using PEG 600 as the cosurfactant revealed a larger ME region area in the diagrams compared to other cosurfactants. Therefore, five SMEPMS formulations with cosurfactant of PEG 600 were selected as a template for preparing FFB nanoparticulates (the exact locations of these five SMEPMS formulations in the phase diagram 


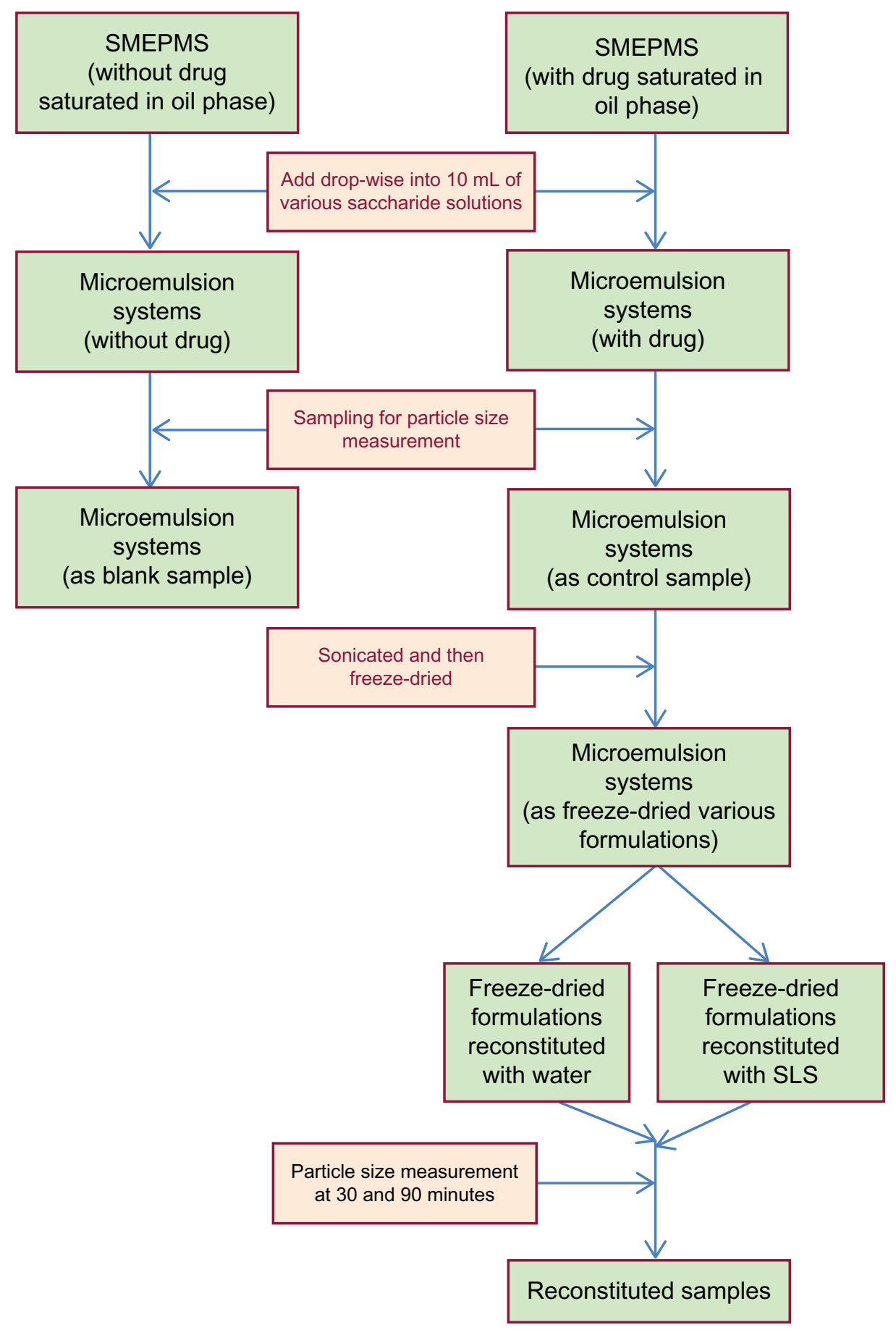

Figure I The scheme of SMEPMS preparation and characterizations.

Abbreviations: SLS, sodium lauryl sulfate; SMEPMS, self-microemulsifying premicroemulsion systems.

are illustrated in Figure 3) and we chose five formulation points (ratio of butyl lactate, Tween 80 , and PEG 600). The composition and dilution status with water (ME or E) for these five SMEPMS formulations are listed in Table 1. For comparison, all three cosurfactants, PEG $600\left(\mathrm{~A}_{\mathrm{pe}}-\mathrm{E}_{\mathrm{pe}}\right)$, propanol $\left(A_{p}-E_{p}\right)$, and ethanol $\left(A_{e}-E_{e}\right)$, were included in the following study of preparing nanoparticulates of FFB, and their compositions and dilution status with water are also listed in Table 1.

Preparation and size analysis of nanoparticles using SMEPMS as templates

It was preliminarily found that, when simply using water as the dilution phase, the reconstitution of FD powder was unable 

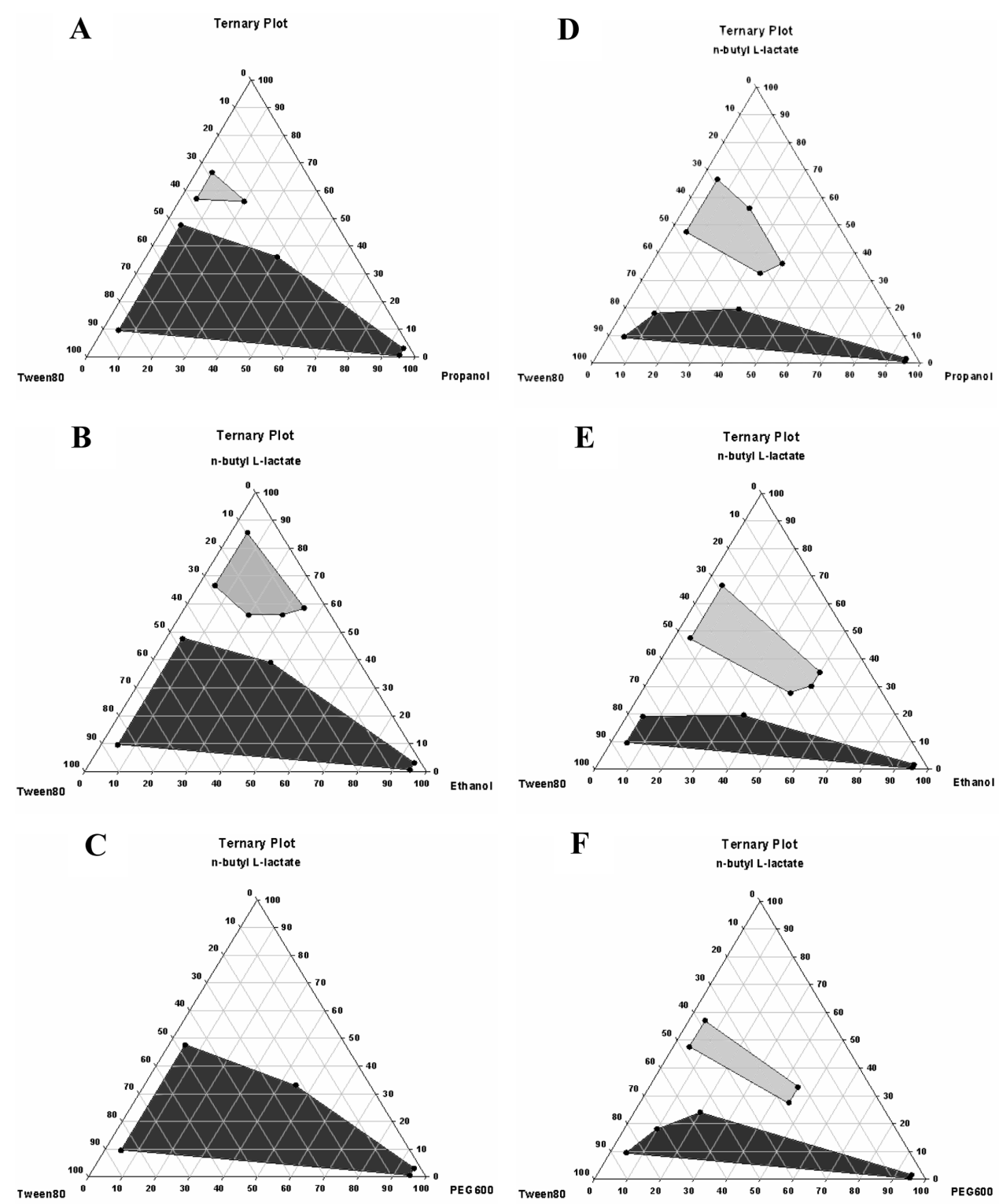

Figure 2 Phase diagrams of butyl lactate/surfactant (Tween 80)/cosurfactants.

Notes: Phase diagrams of butyl lactate/surfactant (Tween 80)/cosurfactants (including propanol [A and D], ethanol [B and E], and PEG 600 [C and F]) without (A-C) and with the addition of fenofibrate (D-F). Black area indicates the microemulsion region, while gray indicates the emulsion region.

to form the desired nanoparticle size regardless of composition. Therefore, the effects of various saccharide solutions (L, lactose; M, mannitol; G, glucose; S, sucrose; or T, trehalose) at $5 \%(50)$ or $10 \%$ concentration (100) as anticaking agents present in the dilution aqueous phase during FD were compared. Results of five SMEPMS formulations as templates using PEG $600\left(\mathrm{~A}_{\mathrm{pe}}-\mathrm{E}_{\mathrm{pe}}\right)$, propanol $\left(\mathrm{A}_{\mathrm{p}}-\mathrm{E}_{\mathrm{p}}\right)$, or ethanol $\left(\mathrm{A}_{\mathrm{e}}-\mathrm{E}_{\mathrm{e}}\right)$ as the cosurfactant are illustrated in Tables $2-4$, respectively (exemplifying those reconstituted in the $0.5 \%$ SLS solution). Table 2 shows that particle sizes of the blank and control were within the nanosize range when PEG 600 was the cosurfactant. Hence, these five SMEPMS formulations selected as templates were capable of forming ME after being diluted with water; however, the particle sizes increased after diluting with all saccharide solutions (under the "BFD" column) compared to those diluting with water (as the control). Particle sizes also tended to increase with higher concentrations of saccharide in the dilution, especially for dilution of $10 \%$ lactose solution (L100). Nevertheless, most of those still maintained their particle sizes within the nanosize range, except when diluted with the $10 \%$ lactose solution (excluding $\mathrm{D}_{\mathrm{pe}}$ ) and with $\mathrm{A}_{\mathrm{pe}}$ diluted with the $10 \%$ glucose solution.

After the process of freeze-drying, these FD FFB samples were reconstituted in either deionized (DI) water or a $0.5 \%$ SLS solution, and their particle sizes were measured at 30 and 90 minutes after reconstitution. The transition time (TT) 


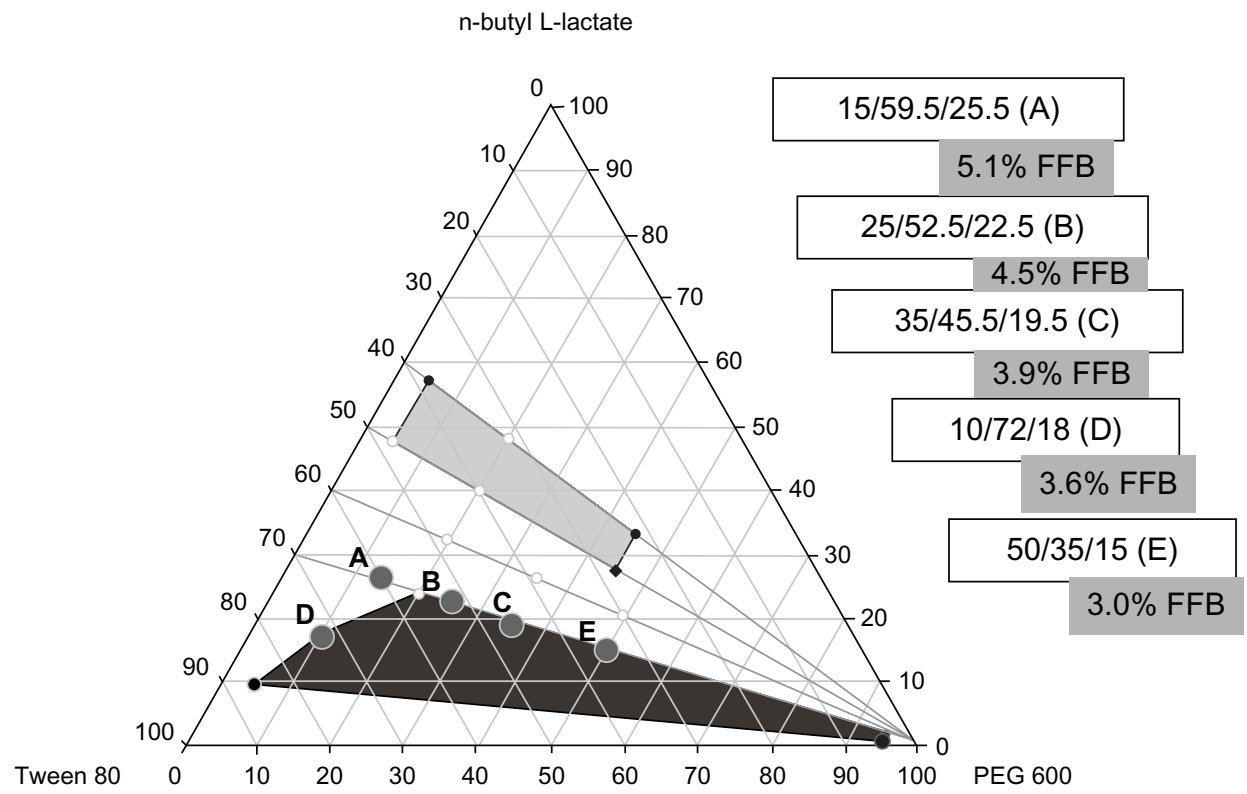

Figure 3 Phase diagrams of butyl lactate/Tween 80/PEG 600 with 100\% FFB loaded in butyl lactate.

Notes: Five points of SMEPMS formulation (A-E).

Abbreviations: FFB, fenofibrate; SMEPMS, self-microemulsifying premicroemulsion systems.

elapsed before the appearance of turbidity after reconstitution was recorded and designated TT. TT means that, after reconstitution, the time when the nanosized FD FFB (particle size $<250 \mathrm{~nm}$ ) precipitated or recrystallized is long enough to turn the solution turbid. All these results are respectively given under the columns of AFD30 m, AFD90 m, and TT in Table 2. TT of $>0.0$ indicated the formation of FFB nanoparticles after FD, and a longer TT implied a more stable FFB nanoparticle after dispersion. TT data in Table 2 demonstrate that SMEPMS using PEG 600 as the cosurfactant could be utilized as templates to form FFB nanoparticles in the five saccharide solutions, and nanoparticles were recovered after FD. Furthermore, reconstitution of the FD FFB nanoparticles produced in these five saccharide solutions in the $0.5 \%$ SLS solution led to longer TT compared to that in DI water, which indicates that the addition of SLS may have had a solubilization

Table I Compositions of various formulations for SMEPMS

\begin{tabular}{|c|c|c|c|c|c|c|c|c|}
\hline \multirow[t]{2}{*}{ Code } & \multirow{2}{*}{$\frac{\text { Oil phase }}{\text { BL }}$} & \multicolumn{2}{|l|}{ Surfactant } & \multicolumn{3}{|c|}{ Cosurfactant } & \multirow[t]{2}{*}{ Oil/s $S_{\text {mix }}$} & \multirow{2}{*}{$\begin{array}{l}\text { Dilution } \\
\text { phase }\end{array}$} \\
\hline & & Tween 20 & Tween 80 & I-Propanol & Ethanol & PEG 600 & & \\
\hline$\overline{A_{p}}$ & 255 & & 595 & 150 & & & 0.34 & $\mathrm{E}$ \\
\hline$B_{p}$ & 225 & & 525 & 250 & & & 0.29 & $\mathrm{E}$ \\
\hline$C_{p}^{p}$ & 195 & & 455 & 350 & & & 0.24 & ME \\
\hline$D_{p}$ & 180 & & 720 & 100 & & & 0.22 & ME \\
\hline$E_{p}$ & 150 & & 350 & 500 & & & 0.18 & ME \\
\hline$A_{e}^{p}$ & 255 & & 595 & & 150 & & 0.34 & $\mathrm{E}$ \\
\hline$B_{e}$ & 225 & & 525 & & 250 & & 0.29 & $E$ \\
\hline $\mathrm{C}_{\mathrm{e}}$ & 195 & & 455 & & 350 & & 0.24 & ME \\
\hline$D_{e}$ & 180 & & 720 & & 100 & & 0.22 & ME \\
\hline$E_{e}$ & 150 & & 350 & & 500 & & 0.18 & ME \\
\hline$A_{p e}$ & 255 & & 595 & & & 150 & 0.34 & $E$ \\
\hline$B_{p e}$ & 225 & & 525 & & & 250 & 0.29 & ME \\
\hline$C_{p e}$ & 195 & & 455 & & & 350 & 0.24 & ME \\
\hline$D_{p e}$ & 180 & & 720 & & & 100 & 0.22 & ME \\
\hline $\mathrm{E}_{\mathrm{pe}}$ & 150 & & 350 & & & 500 & 0.18 & ME \\
\hline $\mathrm{F}_{\mathrm{pe}}$ & 225 & 525 & & & & 250 & 0.29 & $E$ \\
\hline $\mathrm{G}_{\mathrm{pe}}$ & 195 & 455 & & & & 350 & 0.24 & $\mathrm{E}$ \\
\hline $\mathrm{H}_{\mathrm{pe}}$ & 180 & 720 & & & & 100 & 0.22 & ME \\
\hline
\end{tabular}

Abbreviations: BL, n-butyl L-lactate; E, emulsion; ME, microemulsion; Oil/S mix $^{\prime}$ mixture of surfactant and cosurfactant; SMEPMS, self-microemulsifying premicroemulsion systems. 


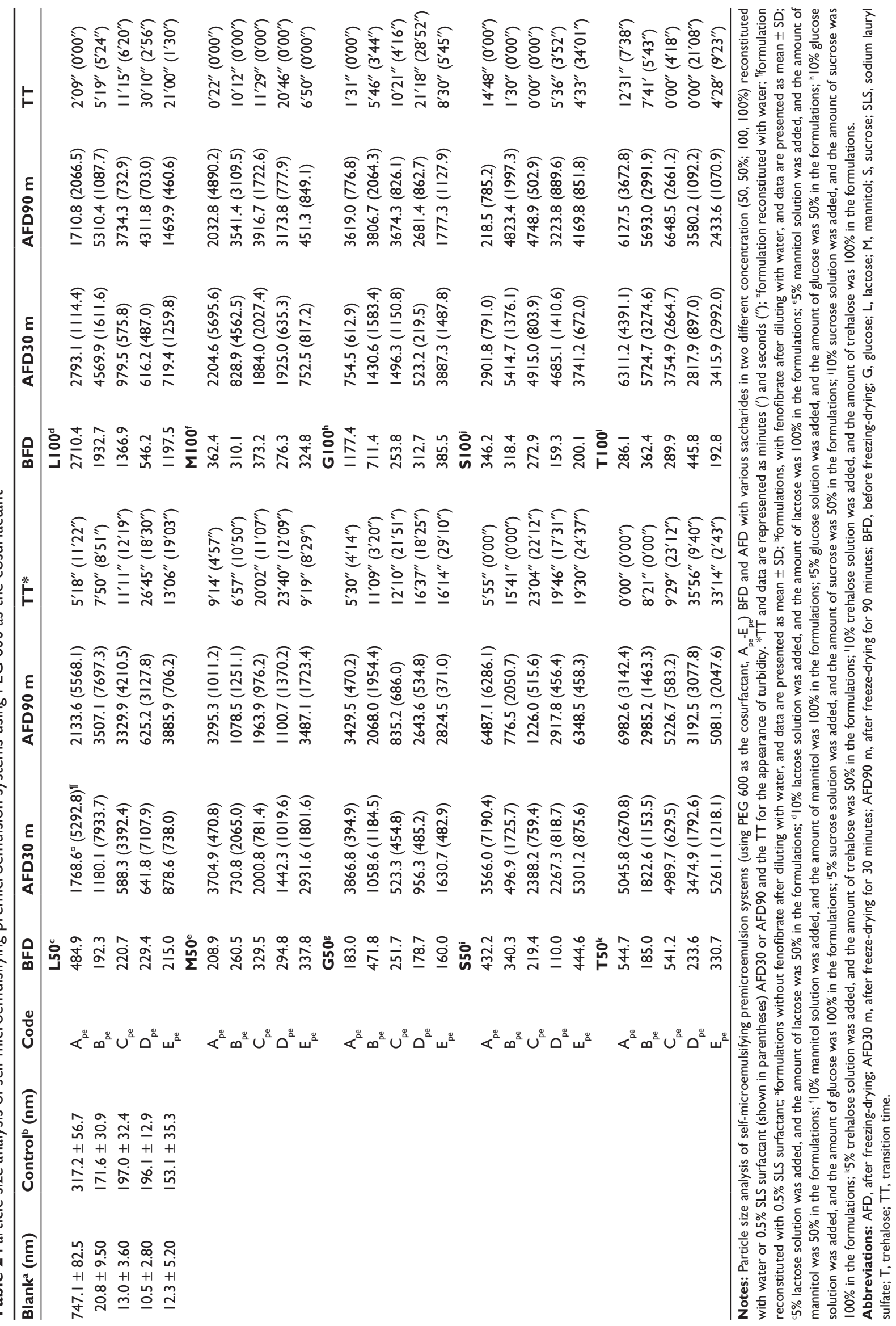









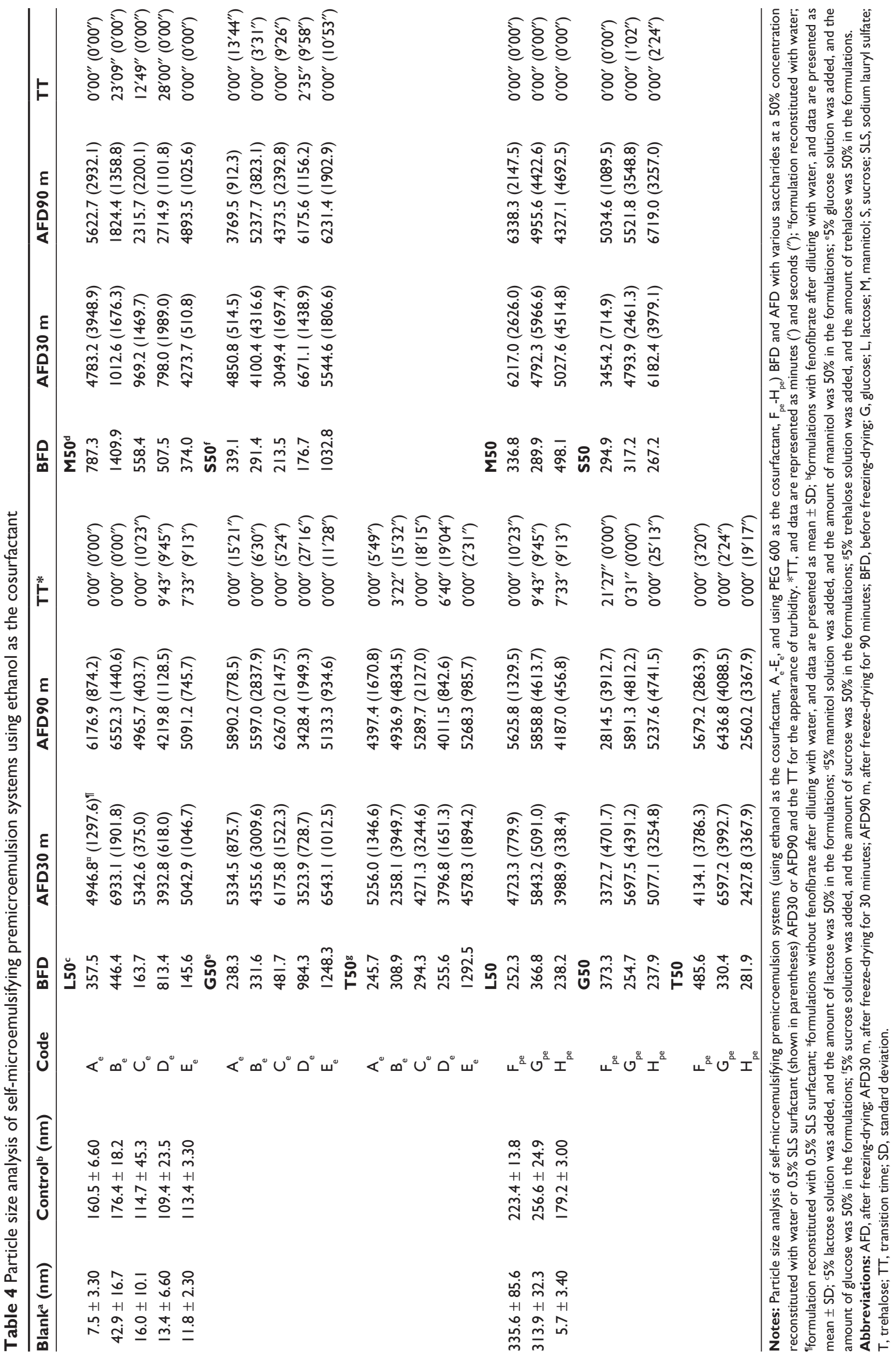


effect on these FFB nanoparticles. Among the five saccharide solutions, the enhanced stability of the resultant FFB nanoparticles in the $5 \%$ glucose solution was the most profound. An increasing concentration of saccharide did not improve the stability of FFB nanoparticles when dispersed in the corresponding solution. Furthermore, it turned out that $0.5 \%$ SLS also maintained FFB nanoparticles within the nanosize range in the reconstitution solution for as long as 90 minutes (marked with deep-colored shading). This stabilization effect was most effective for FFB FD nanoparticles from the 5\% glucose solution using $\mathrm{A}_{\mathrm{pe}}, \mathrm{C}_{\mathrm{pe}}, \mathrm{D}_{\mathrm{pe}}$, and $\mathrm{E}_{\mathrm{pe}}$ SMEPMS as templates (but not $\mathrm{B}_{\mathrm{pe}}$ ) and from the $5 \%$ sucrose solution using $\mathrm{C}_{\mathrm{pe}}, \mathrm{D}_{\mathrm{pe}}$, and $\mathrm{E}_{\mathrm{pe}}$ SMEPMS as templates (but not $\mathrm{A}_{\mathrm{pe}}$ or $\mathrm{B}_{\mathrm{pe}}$ ). This phenomenon might be explained by the $\mathrm{C}_{\mathrm{pe}}, \mathrm{D}_{\mathrm{pe}}$, and $\mathrm{E}_{\mathrm{pe}}$ SMEPMS formulations with PEG 600 as the cosurfactant forming MEs after dilution with the aqueous phase as indicated in Table 1, where $\mathrm{A}_{\mathrm{pe}}$ and $\mathrm{B}_{\mathrm{pe}}$ SMEPMS formulations were only able to form Es. It was concluded that using SMEPMS formulations with PEG 600 as the cosurfactant as templates was able to produce FFB nanoparticles in the presence of a suitable saccharide as an anticaking agent and FD process.

Tables 3 and 4 further list the particle sizes of the blank and control using five SMEPMS formulations with propanol or ethanol as the cosurfactant, respectively. After diluting these five SMEPMS formulations with either propanol or ethanol as the cosurfactant in all saccharide solutions, TT values of the FD FFB samples reconstituted in either DI water or the $0.5 \%$ SLS solution and their particle sizes, measured at 30 and 90 minutes after reconstitution, are also shown in Tables 3 and 4. Table 4 gives the results of using three SMEPMS formulations ( $F$ versus A, $G$ versus B, and $H$ versus $C$ ) with Tween 20 as the surfactant and PEG 600 as the cosurfactant as templates to produce FFB nanoparticles. Similar to the five SMEPMS formulations with PEG 600 as the cosurfactant, nanoemulsions formed from the blank and control group, and the particle sizes still fell within the nanosize range after dilution with most of the saccharide solutions before FD. However, TT values in Tables 3 and 4 indicate that there were quite a few cases of FD FFB particles remaining as nanoparticles after FD. Particle sizes of most of those FD FFB particles after reconstitution in either DI water or the $0.5 \%$ surfactant did not form nanoparticles. It was concluded that SMEPMS formulations with Tween 80 as the surfactant and PEG 600 as the cosurfactant were optimal templates to produce FFB nanoparticles.

\section{Characterization of FD nanosized FFB}

Figure 4 shows SEM images of $\mathrm{D}_{\mathrm{pe}}$ with four saccharide solutions as the solid carrier. $\mathrm{D}_{\mathrm{pe}}$ with $5 \%$ mannitol and lactose

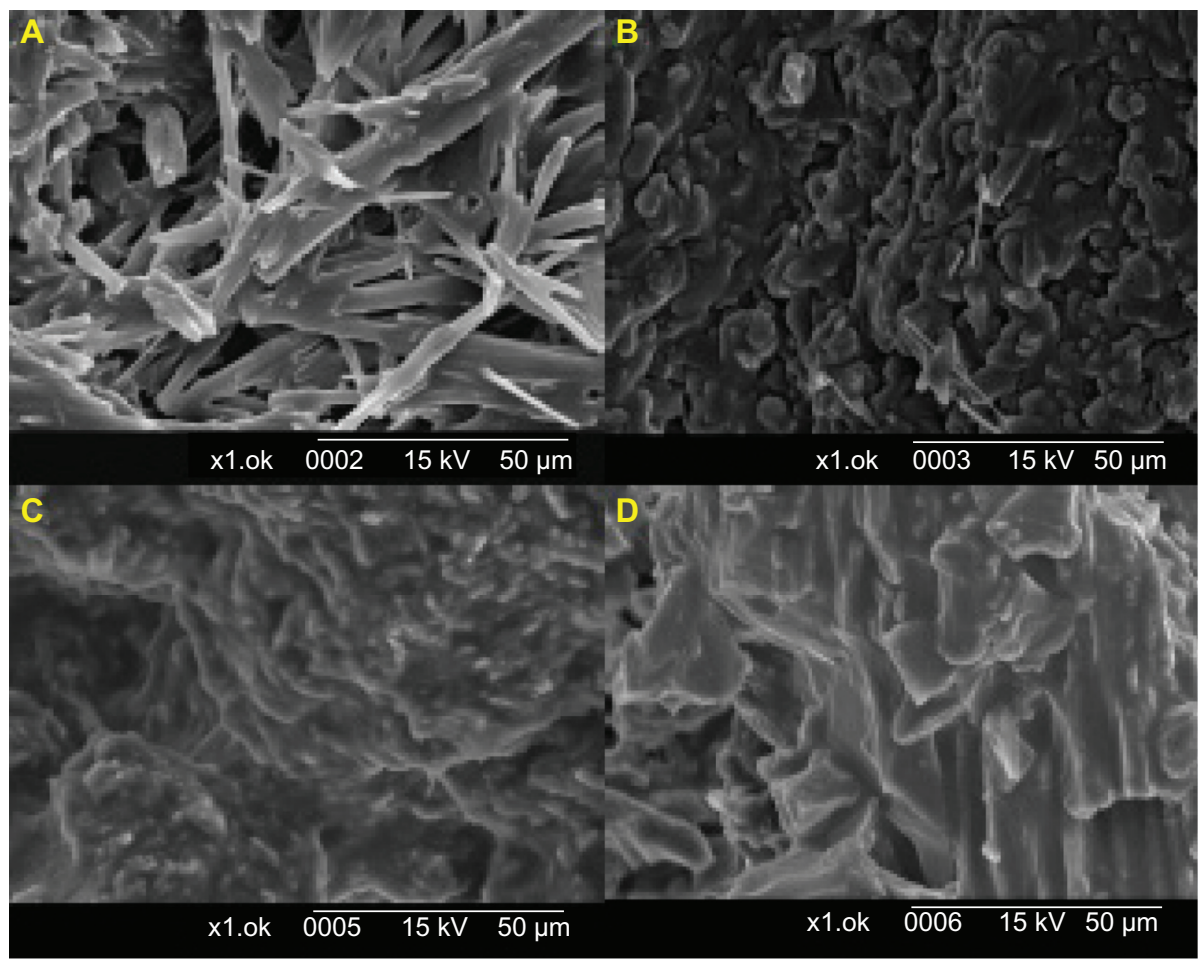

Figure 4 Scanning electron microscopic images of freeze-dried formulations of $D_{\mathrm{pe}}$.

Notes: (A) 5\% mannitol, (B) 10\% mannitol, (C) 10\% lactose, and (D) 10\% trehalose solutions (Zoom ratio: 1000). 
(data not shown) had fewer amorphous forms, whereas that with $10 \%$ mannitol had fewer crystalline needles and better particle dispersion. The presence of saccharide might inhibit the formation of drug crystals, and the extent of inhibition of the lattice arrangement of drug crystals with various saccharides differed. The formulation with trehalose caused columnar crystals and poor redispersibility. Results of powder X-ray diffractometry were consistent with those of the particle size analysis. The peak intensity of FD nanosized FFB was much lower than that of the pure saccharide solution as shown in Figure 5, and a rearrangement of the lattice structure during the process of dilution might have contributed. Compared to the peak of FFB, the lower peak intensity of FD nanosized FFB indicates that the FFB formed amorphous nanoparticles through the use of surfactants, cosurfactants, and the added saccharides.
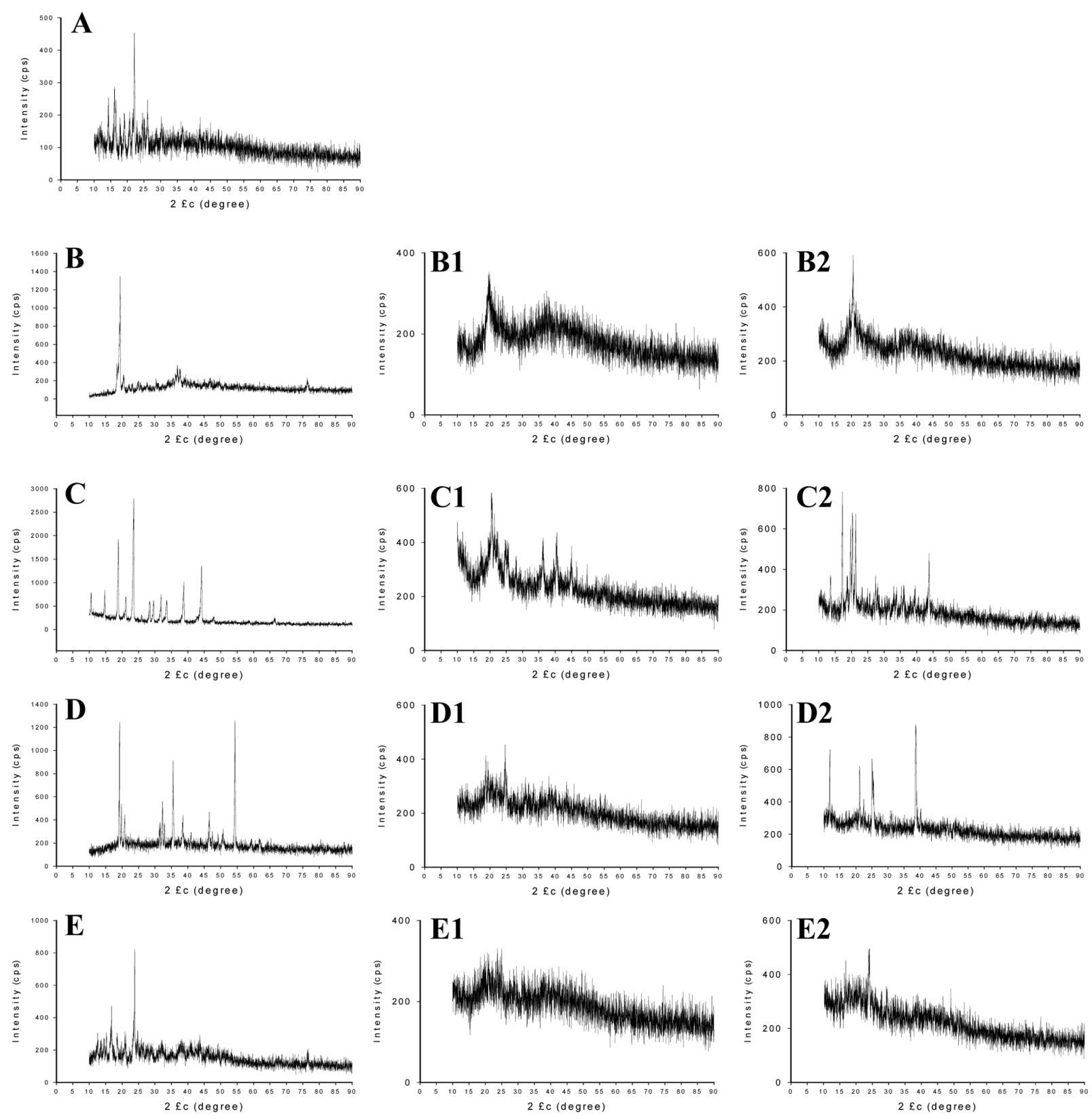

Figure 5 Powder X-ray diffractometry.

Notes: (A) fenofibrate only; (B) lactose only; $D_{\text {pe }}$ freeze-dried with a $5 \%$ (BI) or 10\% (B2) lactose solution; (C) mannitol only; $D_{\text {pe }}$ freeze-dried with a $5 \%$ (CI) or 10\% (C2) mannitol solution; (D) sucrose only; $D_{p e}$ freeze-dried with a $5 \%$ (DI) or 10\% (D2) sucrose solution; (E) trehalose only; $D_{p e}$ freeze-dried with a $5 \%$ (EI) or I0\% (E2) trehalose solution. 


\section{Conclusion}

FFB saturated in n-butyl L-lactate, Tween 80, and a number of cosurfactants (ethanol, 1-propanol, and PEG 600) and then diluted with saccharide solutions prior to the FD process was developed. It was concluded that, by using SMEPMS formulations with PEG 600 as the cosurfactant, FFB nanoparticles were able to be produced, and FD in the presence of a suitable saccharide as an anticaking agent was necessary to maintain the particle size stability of the reconstituted powder. Our studies indicate that the potential utilization of SMEPMS for the delivery of poorly soluble drugs, such as FFB, improve its bioavailability; furthermore, the use of lyophilization and the addition of saccharide made an FFB oral solid nanoparticle dosage form with easy administration. The SMEPMS templates establish a database about monodispersed sizes of nanoparticles (inorganic or organic) by varying the size of the $\mathrm{ME}$ droplet radius, allowing one to use the information to design orally active dosage form.

\section{Disclosure}

The authors report no conflicts of interest in this work.

\section{References}

1. Lipinski CA, Lombardo F, Dominy BW, Feeney PJ. Experimental and computational approaches to estimate solubility and permeability in drug discovery and development settings. Adv Drug Deliv Rev. 2000;46(1-3): 3-26.

2. Pouton $\mathrm{CW}$. Formulation of poorly water-soluble drugs for oral administration: physicochemical and physiological issues and the lipid formulation classification system. Eur J Pharm Sci. 2006;29(3-4): 278-287.

3. Munoz A, Guichard JP, Reginault P. Micronised fenofibrate. Atherosclerosis. 1994;110 Suppl:S45-S48.

4. Hoar TP, Schulman JH. Transparent water-in-oil dispersions: The oleopathic hydro-micelle. Nature. 1943;152(3847):102-103.

5. Spernath A, Aserin A. Microemulsions as carriers for drugs and nutraceuticals. Adv Colloid Interface Sci. 2006;128-130:47-64.

6. Stuchlík M, Zák S. Lipid-based vehicle for oral drug delivery. Biomed Pap Med Fac Univ Palacky Olomouc Czech Repub. 2001;145(2):17-26.

7. Pouton CW. Lipid formulations for oral administration of drugs: nonemulsifying, self-emulsifying and 'self-microemulsifying' drug delivery systems. Eur J Pharm Sci. 2000;11 Suppl 2:S93-S98.

8. Constantinides PP. Lipid microemulsions for improving drug dissolution and oral absorption: physical and biopharmaceutical aspects. Pharm Res. 1995;12(11):1561-1572.
9. Khoo SM, Humberstone AJ, Porter CJH, Edwards GA, Charman WN. Formulation design and bioavailability assessment of lipidic selfemulsifying formulations of halofantrine. Int J Pharm. 1998;167(1-2): 155-164.

10. Friman S, Bäckman L. A new microemulsion formulation of cyclosporin: pharmacokinetic and clinical features. Clin Pharmacokinet. 1996;30(3):181-193.

11. Kim JY, KuYS. Enhanced absorption of indomethacin after oral or rectal administration of a self-emulsifying system containing indomethacin to rats. Int J Pharm. 2000;194(1):81-89.

12. Kang BK, Lee JS, Chon SK, et al. Development of self-microemulsifying drug delivery systems (SMEDDS) for oral bioavailability enhancement of simvastatin in beagle dogs. Int J Pharm. 2004;274(1-2):65-73.

13. Chen Y, Li G, Wu X, et al. Self-microemulsifying drug delivery system (SMEDDS) of vinpocetine: formulation development and in vivo assessment. Biol Pharm Bull. 2008;31(1):118-125.

14. Woo JS, Song YK, Hong JY, Lim SJ, Kim CK. Reduced food-effect and enhanced bioavailability of a self-microemulsifying formulation of itraconazole in healthy volunteers. Eur J Pharm Sci. 2008;33(2): $159-165$.

15. Fatouros DG, Nielsen FS, Douroumis D, Hadjileontiadis LJ, Mullertz A. In vitro-in vivo correlations of self-emulsifying drug delivery systems combining the dynamic lipolysis model and neuro-fuzzy networks. Eur J Pharm Biopharm. 2008;69(3):887-898.

16. Cooney GF, Jeevanandam V, Choudhury S, Feutren G, Mueller EA, Eisen HJ. Comparative bioavailability of neoral and sandimmune in cardiac transplant recipients over 1 year. Transplant Proc. 1998;30(5): 1892-1894.

17. Venkatesh G, Majid MIA, Mansor SM, Nair NK, Croft SL, Navaratnam V. In vitro and in vivo evaluation of self-microemulsifying drug delivery system of buparvaquone. Drug Dev Ind Pharm. 2010;36(6):735-745.

18. Nazzal S, Khan MA. Controlled release of a self-emulsifying formulation from a tablet dosage form: stability assessment and optimization of some processing parameters. Int J Pharm. 2006;315(1-2):110-121.

19. Trotta M, Gallarate M, Pattarino F, Morel S. Emulsions containing partially water-miscible solvents for the preparation of drug nanosuspensions. J Control Release. 2001;76(1-2):119-128.

20. Trotta M, Gallarate M, Carlotti ME, Morel S. Preparation of griseofulvin nanoparticles from water-dilutable microemulsions. Int J Pharm. 2003;254(2):235-242.

21. Clary JJ, Feron VJ, van Velthuijsen JA. Safety assessment of lactate esters. Regul Toxicol Pharmacol. 1998;27(2):88-97.

22. Wei L, Sun P, Nie S, Pan W. Preparation and evaluation of SEDDS and SMEDDS containing carvedilol. Drug Dev Ind Pharm. 2005; 31(8):785-794.

23. Patel AR, Vavia PR. Preparation and in vivo evaluation of SMEDDS (self-microemulsifying drug delivery system) containing fenofibrate. AAPS J. 2007;9(3):E344-E352.

24. Shen H, Zhong M. Preparation and evaluation of self-microemulsifying drug delivery systems (SMEDDS) containing atorvastatin. J Pharm Pharmacol. 2006;58(9):1183-1191.

25. Kogan A, Aserin A, Garti N. Improved solubilization of carbamazepine and structural transitions in nonionic microemulsions upon aqueous phase dilution. J Colloid Interface Sci. 2007;315(2):637-647.
International Journal of Nanomedicine

\section{Publish your work in this journal}

The International Journal of Nanomedicine is an international, peerreviewed journal focusing on the application of nanotechnology in diagnostics, therapeutics, and drug delivery systems throughout the biomedical field. This journal is indexed on PubMed Central, MedLine, CAS, SciSearch ${ }^{\circledR}$, Current Contents ${ }^{\circledR} /$ Clinical Medicine,

\section{Dovepress}

Journal Citation Reports/Science Edition, EMBase, Scopus and the Elsevier Bibliographic databases. The manuscript management system is completely online and includes a very quick and fair peer-review system, which is all easy to use. Visit http://www.dovepress.com/ testimonials.php to read real quotes from published authors. 